\title{
Notas preliminares sobre os morcegos do Parque Nacional da Amazônia (Médio Tapajós)
}

\author{
Nelio Roberto dos Reis ( $\left.{ }^{(}\right)$ \\ Herbert O. R. Schubart $\left({ }^{* *}\right)$
}

Resumo

Estudo sobre morcegos realizado no Parque Nacional da Amazônia, localizado em terras do Pará e Amazonas com 1.000 .000 de hectares. Cento e trinta e três individuos de 17 espécies de 5 familias foram cap. turados, sendo que 12 espécies pertencem à familia Phillostomidae. O gênero Carollia é o mais comum. Conclui-se que a coexistência de muitas espécies em um único local é possível devido à diversificaçāo do hábito alimentar. Morcegos frugivoros foram os mais coletados, mas foram encontrados ainda insetivoros. nectarivoros e hematófagos. Foi feita uma comparaçäo da fauna de morcegos entre Manaus, Parque Nacional da Amazônia e Belém: somente $11 \%$ das espécies são comuns aos 3 lugares, sendo que a fauna do Parque mostra maior afinidade com a de Manaus.

\section{INTRODUÇÃo}

O Parque Nacional da Amazônia, com 1 milhão de hectares, na grande maioria ainda fioresta primária, à margem esquerda do rio Tapajós, Município de Itaituba, abrange terras dos Estados do Amazonas e Pará (Fig. 1) (aproximadamente $3^{\circ} 50^{\prime}, 5^{\circ} \mathrm{S}$ e $56^{\circ} 15^{\prime}, 57^{\circ} 32^{\prime} \mathrm{W}$ ). A temperatura média anual varia entre $24^{\circ} \mathrm{C}$ e $26^{\circ} \mathrm{C}$, e a região apresenta um índice pluviométrico anual de aproximadamente $1.754 \mathrm{~mm}$. A região portanto, enquadra-se dentro de um clima quente e úmido (IBDF, 1978). O obje. tivo dentro da área manejável do Parque $\mathrm{Na}$ cional da Amazônia é de fazer um levantamento preliminar das espécies de morcegos existentes, colher dados sobre sua ecologia e distribuição, e fazer um estudo comparativo com os morcegos da região de Manaus e Be. lém, para se ter uma noção preliminar sobre a zoogeografia deste grupo na Amazônia.
MÉToDos

As técnicas de capturas foram adaptadas das de Greenhall \& Paradiso (1968) e Taddei (1973).

Oitenta e seis horas de capturas intensivas foram realizadas durante o mês de agosto de 1978 , normalmente das 18 às 21 horas. horário de maior incidência dos morcegos Portanto, na tabela 2 , onde estes resultados foram tabulados, 9 horas de capturas representam o resultado de 3 dias de coletas. Exceto no Uruá (sede do Parque) onde além das coletas periódicas das 18 às 21 horas, foram feitas coletas intensivas durante 3 noites. Ainda foram feitas capturas diurnas nos lugares de repouso dos morcegos, assim como bueiros de estradas, ocos de árvores e locas de pedras, cercando com redes estes locais e posteriormente espantando os animais.

Foram usadas durante as capturas de vôo livre entre 120 a $180 \mathrm{~m}^{2}$ de redes para aves (mist net). As redes eram assim distribuídas: $100 \mathrm{~m}^{2}$ armados permanentemente no Uruá e o restante, que variava entre 20 a $80 \mathrm{~m}^{2}$ de redes foram armadas alternadamente em 10 lugares diferentes, conforme mostra a figura 2: $1-\mathrm{Km} 60$, ramal do morro da Terra Preta; $2-\mathrm{Km}$ 65, ramal da Anta; $3-\mathrm{Km} 65$, área residencial do Uruá; $4-\mathrm{Km} 66$, bueiro de estrada : 5 - cachoeira do Uruá; 6 - ilha do rio Tapajos, entre o povoado de Pimental e a sede do P.N. da Amazônia; $7-\mathrm{Km}$ 68, ramal do Sayta; $8-\mathrm{Km} \mathrm{75}$, ramal de mata primária; $9-\mathrm{Km} \mathrm{80}$, igarapé Mambuaí; $10-$ Km 140, ramal de mata primária. Todos estes locais estão dentro da área manejável do Par-

(*) - Universidade Estaduai de Londrina, Paraná.

(") - Instituto Nacional de Pesquisas da Amazônia, Manaus. 


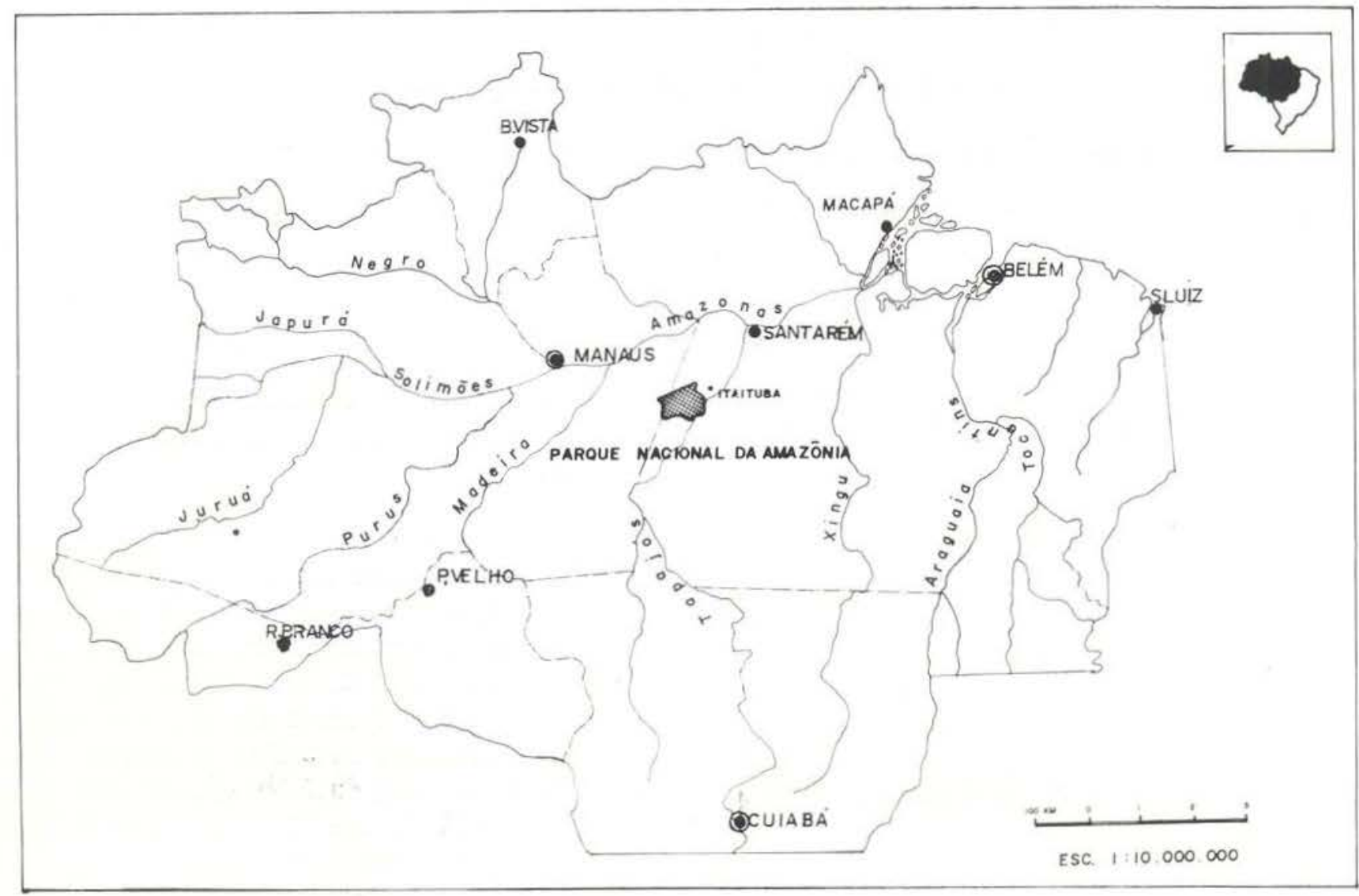

Fig. 1 - Localizaçăo do Parque Nacional da Amazônia na Amazônia Legal.

que. Estes marcos de quilometragem são da rodovia Transamazônica sentido Itaituba-Jacareacanga, margem esquerda do rio Tapajós.

Dois exemplares de cada espécie de morcego foram sacrificados com eter e fixados com formol a $10 \%$ e preservados em álcool \$2\% para identificação posterior. Os critérios de identificação foram os de Goodwin \& Greenhall (1961), Husson (1962) e ainda Vizotto \& Taddei (1973). Alguns exemplares foram identificados pelos professores Ivam Sazima e Wilson Uieda da Universidade Estadual de Campinas.

Parte dos morcegos preservados se encontra depositada na coleção do Departamen. to de Zoologia da Universidade Estadual de Campinas e parte na coleção do Departamento de Ciências Biológicas (zoologia) da Faculdade de Filosofia, Ciências e Letras de S. J. do Rio Preto. Posteriormente, estes 133 espécimes do P. N. da Amazônia, foram compara- dos com 1177 exemplares coletados nos arredores de Manaus e com 1157 exemplares coletados nos arredores de Belém. As referências das coletas de Manaus são: Reis \& Mok (1979), Pirlot (1972) e ainda por dados pessoais não publicados. Sobre as coletas realizadas em Belém as referências são de Handley (1967).

\section{RESUltados E DISCUSSÃO}

Em apenas 86 horas de capturas, em um único mês, 17 espécies de morcegos foram capturados na parte manejável do Parque $\mathrm{Na}$. cional da Amazônia. Esta parte corresponde a menos de $1 / 5$ da área de 1 milhão de hectares. Fazendo-se coletas periódicas durante 1 ano, poder-se-ia chegar a mais de 50 espécies. Isto porque existem evidências de que algumas espécies realizam migrações locais, mais por condições alimentares do que por fa- 
TABELA 1 - Hábito alimentar e distribuição dos morcegos coletados no Parque Nacional da Amazônia

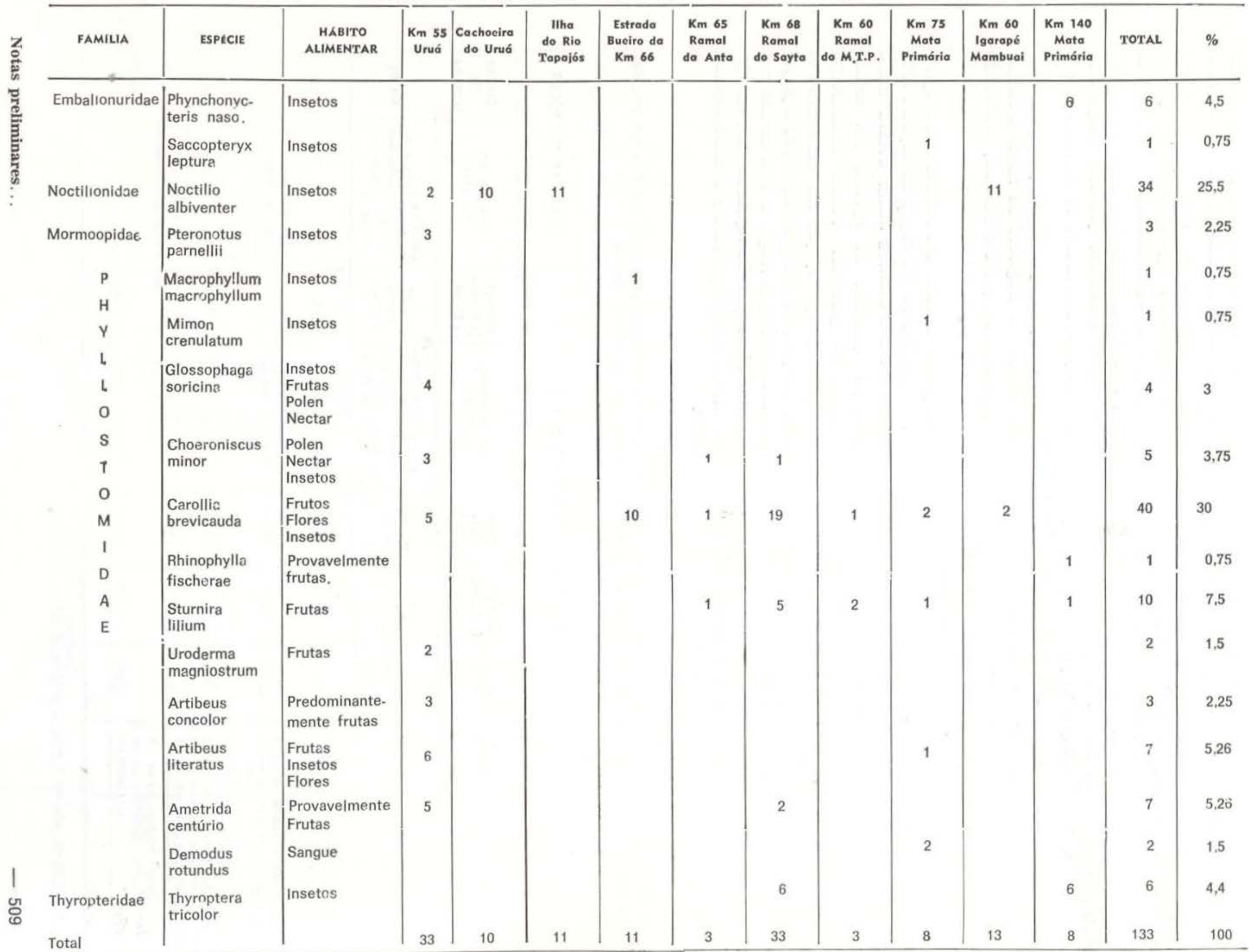


tores climáticos (Taddei, 1973). Esta grande diversidade de morcegos também ocorre em outros locais da Amazônia; isto já foi verificado por nós dentro do Município de Manaus, em 1977 e 1978 (não publicado), e por Handley (1967) que coletou um razoável número de espécies em Belém em um tempo extremamente curto. A nossa hipótese para coexistência de tantas espécies é a grande diversificação da oferta de alimentos. Em uma zona temperada, os morcegos em sua grande maioria são insetivoros: nos trópicos são encontrados morcegos de todos os hábitos alimentares.

Os morcegos frugivoros foram obtidos em maior número dentro do Parque (Tabela 1). Eles são propriamente os habitantes dos trópicos, especialmente em regióes de florestas, onde pelo menos algumas espécies de frutas estão em produção em cada época do ano. Dentro deste grupo dos frugívoros (Phyllostomidae), encontramos desde o pequeno Rhynophylla fischerae, com aproximadamente $32 \mathrm{~mm}$ de antebraço, até o enorme Artibeus litura- ius, com até $75 \mathrm{~mm}$ de antebraço. Ainda nesta tamilia, foram encontrados morcegos insetívoros e nectarivoros. A maıor concentraçao de frugivoros, especialmente do gënero Carollia e Sturnira, foi encontrada no ramal do Sayta, sítio abandonado com centenas de pés de bananeira e mamão, que era antiga área cultivável para o consumo de Itaituba.

Com a implantação do Parque, o sítio foi abandonado. O frugivoro de maior distribuição dentro do Parque é o Carollia, encontrado em 7 dos 10 lugares coletados.

A grande maioria das fêmeas dos frugivoros, e de todos os morcegos da espécie Artibeus lituratus e Sturnira lilium estava nesta época (2. quinzena de agosto) em estado adiantado de gravidez. Foram encontrados ainda Glossophaginae, que são extremamente adaptados para se alimentar de néctar e pólen, apresentando língua longa, e com cerdas nas extremidades e que são representados no Parque pelo Glossophaga sorina e Choeroniscus minor.

TABELA 2 - Esforço de captura, expresso em $\mathrm{m}^{2}$ de rede por horas de exposição, empregado nas diversas estaçōes de coleta, e número de espécies e individuos coletados, excetuando-se as coletas diurnas.

\begin{tabular}{|c|c|c|c|c|c|c|c|c|c|}
\hline & \multicolumn{7}{|c|}{ COLETAS NOTURNAS } & \multicolumn{2}{|c|}{ COLETAS DIURNAS } \\
\hline & \multicolumn{2}{|c|}{ MATA PRIMÁRIA } & \multirow{2}{*}{ 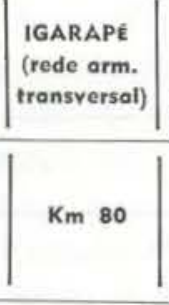 } & \multirow{2}{*}{$\begin{array}{c}\begin{array}{c}\text { ÁREA } \\
\text { RESI- } \\
\text { DENCIAL }\end{array} \\
\text { Km } 65 \\
\text { URUÁ }\end{array}$} & \multicolumn{2}{|c|}{$\begin{array}{c}\text { (mato secundaria) } \\
\text { CAPOEIRA }\end{array}$} & \multirow{2}{*}{\begin{tabular}{|}
$\left|\begin{array}{c}\text { Plantaçăo } \\
\text { de Banana } \\
\text { e Mamäo }\end{array}\right|$ \\
$\mid \begin{array}{c}\text { Km } 68 \\
\text { Ramal } \\
\text { do Sayta }\end{array}$
\end{tabular}} & $\begin{array}{l}\text { ILHA DO RIO } \\
\text { TAPASOS }\end{array}$ & $\begin{array}{c}\text { BUEIRO } \\
\text { DE } \\
\text { ESTRADA }\end{array}$ \\
\hline & $\mathrm{Km} 75$ & $\mathrm{Km} 140$ & & & $\begin{array}{c}\text { Km } 65 \\
\text { Ramal } \\
\text { da Anta }\end{array}$ & $\left|\begin{array}{c}\text { Km } \\
\text { mal do } \text { Mor- } \\
\text { ro da Ter- } \\
\text { ra Preta }\end{array}\right|$ & & \begin{tabular}{|c|l|} 
Cochoeira & llho do Ta- \\
Uruá (lo- & pajós (oco \\
cal de & de árvores) \\
pedras) &
\end{tabular} & $\mathrm{Km} 66$ \\
\hline $\begin{array}{c}\text { Tempo de coletas } \\
\text { (horas) }\end{array}$ & $9 \mathrm{~h}$ & $3 \mathrm{~h}$ & $3 \mathrm{~h}$ & $54 \mathrm{~h}$ & $6 h$ & $3 \mathrm{~h}$ & $3 h$ & $2 \mathrm{~h}$ & $2 h$ \\
\hline $\begin{array}{l}\mathrm{m}^{2} \text { de Rede usada } \\
\text { no captura }\end{array}$ & $52 m^{2}$ & $52 m^{2}$ & $40 \mathrm{~m}^{2}$ & $100 \mathrm{~m}^{2}$ & $20 \mathrm{~m}^{2}$ & $80 \mathrm{~m}^{2}$ & $60 \mathrm{~m}^{2}$ & $\begin{array}{l}\text { rede armada em } \\
\text { volta do lugar } \text { de } \\
\text { repouso do mor- } \\
\text { cego. }\end{array}$ & \\
\hline $\begin{array}{l}\text { N. } \cdot \text { de indivíduos } \\
\text { coletados }\end{array}$ & 8 & 8 & 13 & 35 & 3 & 3 & 33 & 11 & 11 \\
\hline N. ${ }^{\circ}$ de espécies & 6 & 3 & 2 & $10^{*}$ & 3 & 3 & 5 & 1 & 2 \\
\hline $\begin{array}{l}\mathrm{N} .{ }^{\circ} \text { de indivíduos/ } \\
\mathrm{m}^{2} / \mathrm{h} .\end{array}$ & 0,017 & 0,051 & 0.108 & 0,006 & 0,025 & 0,012 & 0,183 & - & - \\
\hline
\end{tabular}

(") $-\mathrm{m}^{2}$ de rede em 1 hora $=$ unidade de esforço de captura. 
Um número razoável de morcegos capturados ainda retém, como seus ancestrais, o primitivo hábito de comer insetos. Mesmo çue os insetos não sejam freqüentes em número durante todo o ano, não se acredita que possam chegar a ser um fator limitante. $O$ Noctilio albiventris e o Rhynchonycteris naso, só foram encontrados nas correntes de água. como mostra a figura 3 , ficando desta maneira restrita a sua distribuição. A outra espécie da família Noctilionidae, Noctilio leporinus, o piscívoro verdadeiro da Amazônia, não foi encontrada. Das três espécies de morcegos hematófagos (vampiros) conhecidas, membros da subfamília Desmodontinae, apenas uma está presente no Parque, o Desmodus rotundus. Foi incomum não se ter capturado nenhum membro Molossidae, grande controlador de insetos que é comum em forro de casas abandonadas, ou mesmo em casas habitadas na Amazônia. A maioria das casas do Parque Nacional da Amazônia foi examinada e morcegos desta família não foram encontrados. Em um contato com um engenheiro da firma Rabello, (fir-

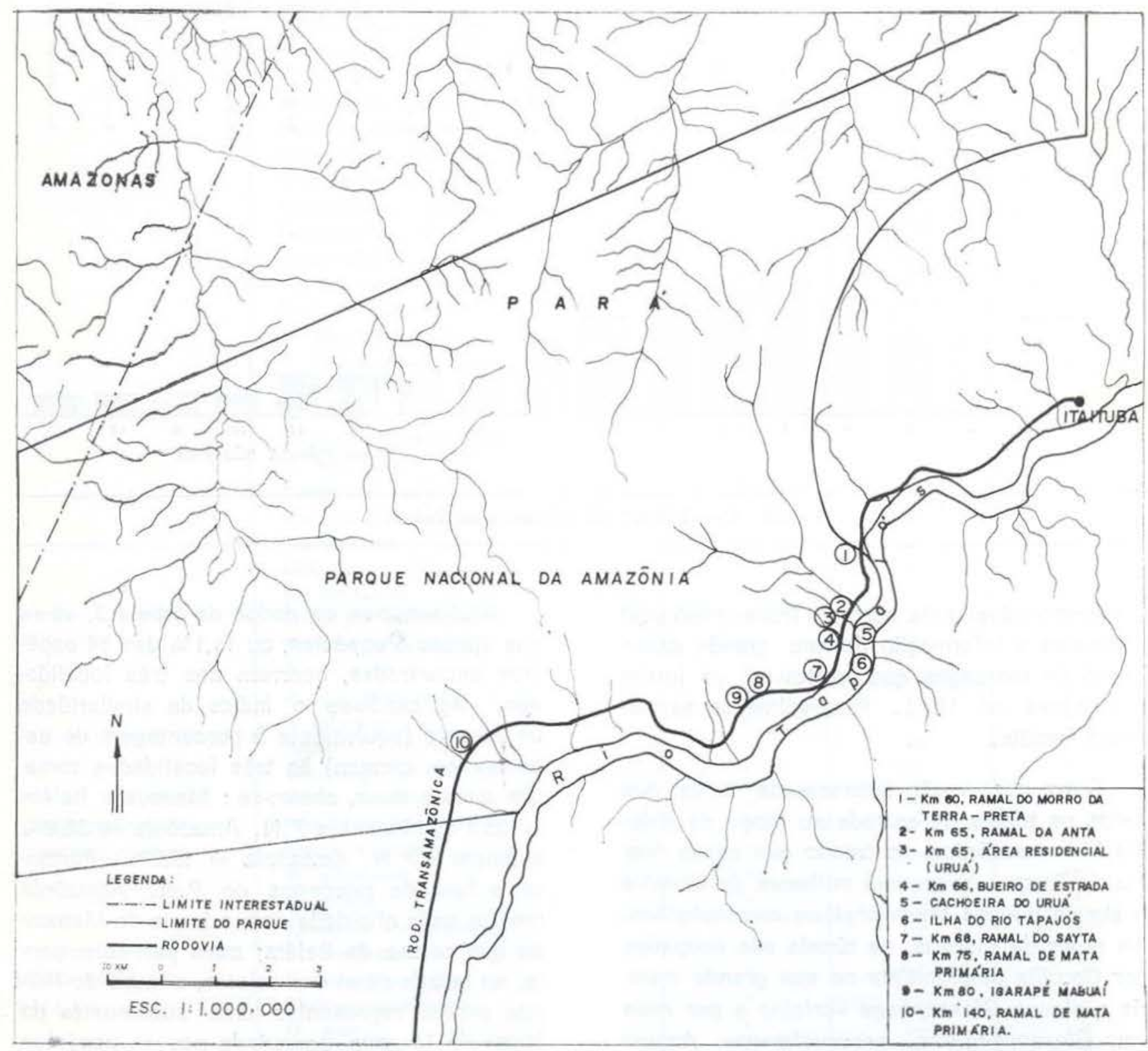

Fig. 2 - Limites do Parque Nacional da Amazônia. 


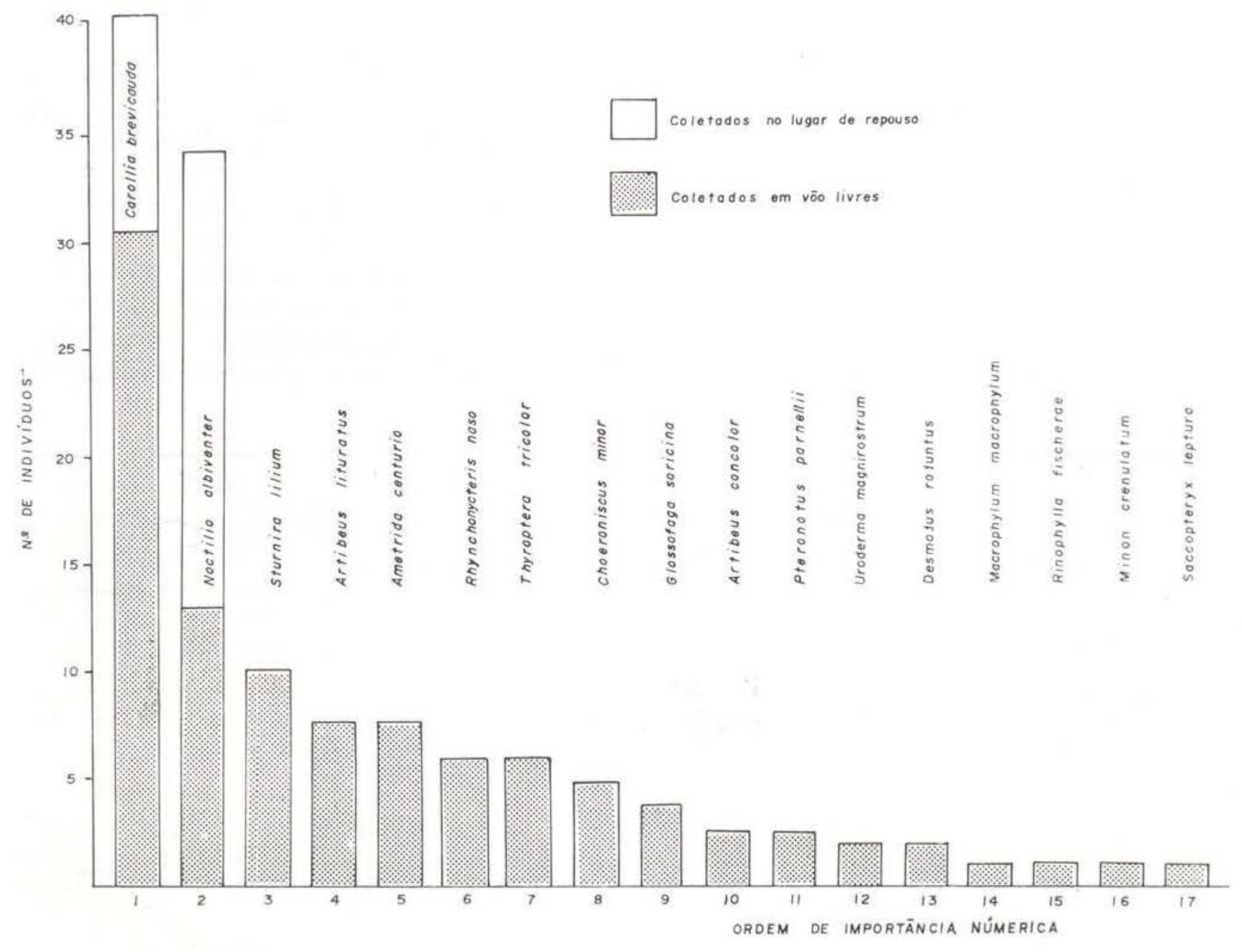

Fig. 3 - Número de morcegos coletados.

ma responsável pela estrada Transamazônica) obteve-se a informação de um grande extermínio de morcegos que habitavam os forros das casas no Uruá. Possivelmente seriam desta familia.

Outra observação interessante é de que todos os túneis de estrada ao longo da rodovia Transamazônica, no trecho que passa dentro do Parque, hospedam milhares de Carollia e alguns poucos Macrophyllum macrophyllum. Ao redor de Manaus, os túneis são ocupados por Carollia perspicillata na sua grande maio. ria e alguns Glossophaga soricina e por mais um Glossophaginae, provavelmente Anoura caudifer, e raramente também por Macrophyllum macrophyllum.
Analisando-se os dados da tabela 3 , vê-se que apenas 6 espécies, ou 11,1\% das 54 espécies encontradas, ocorrem nas três localidades. Aplicando-se o índice de similaridade de Jaccard (equivalente à porcentagem de espécies em comum) às três localidades tomadas duas a duas, obtém-se : Manaus $\times$ Belém $=35,8 \%$, Manaus x P.N. Amazônia $=35,9 \%$ e Belém x P.N. Amazônia $=15,2 \%$. Portanto, a funa de morcegos do P.N. Amazônia mostra mais afinidade com a fauna de Manaus do que com a de Belém; mais particularmente, no estado atual das coletas, a fauna do Parque parece representar uma subamostra da fauna de Manaus, possuindo poucas espécies que lhe sejam próprias. Isto se torna mais claro quando se considera com mais detalhe 
TABELA 3 - Dados sobre a distribuição dos mor cegos coletados no Parque Nacional da Amazônia

\begin{tabular}{|c|c|c|c|c|}
\hline$F A M I L I A S$ & ES PE C IES & MANAUS & $\begin{array}{l}\text { COLETAS } \\
\text { P. N. AMAZ. }\end{array}$ & BELÉM \\
\hline \multirow{44}{*}{$\begin{array}{l}\text { EMBALLONURIDAE } \\
\text { NOCTILIONIDAE } \\
\text { MORMOOPIDAE } \\
\text { PHYLLOSTOMIDAE }\end{array}$} & Rhynchonycteris naso & " & no & $* * 0$ \\
\hline & Saccopteryx bilineata & * & 一 & $\rightarrow$ \\
\hline & S. leptura & $\cdot$ & $\cdots$ & $\rightarrow$ \\
\hline & Cormura brevirortris & $\rightarrow$ & 一 & $\cdots$ \\
\hline & Noctilio albiventris & $\bullet$ & $\cdots$ & \multirow{2}{*}{-} \\
\hline & Pteronotus parnellii & • & $\cdots$ & \\
\hline & Micronycteris minuta & - & - & $\cdots$ \\
\hline & M. nicefori & - & 一 & $\cdots$ \\
\hline & M. sylvestris & 一 & - & $\cdots$ \\
\hline & Macrophyllum macrophyllum & * & $\cdots$ & - \\
\hline & Tonatia bidens & - & 一 & $\cdots$ \\
\hline & T. silvicola & 一 & - & $\cdots$ \\
\hline & Mimon crenulatum & ${ }^{*}$ & $\cdots$ & $\overrightarrow{.}$ \\
\hline & Phyllostomus discolor & $\cdot$ & $\rightarrow$ & $\cdots$ \\
\hline & P. elongatus & 一 & - & $\cdots$ \\
\hline & P. hastatus & $\cdot$ & - & $\cdots$ \\
\hline & Phylloderma stenops & 一 & - & $\cdots$ \\
\hline & Trachops cirrhosus & $\cdot$ & 一 & $\cdots$ \\
\hline & Glossophaga soricina & $\cdot$ & $\cdots$ & $\cdots$ \\
\hline & Lionycteris spurrelli & - & 一 & $\cdots$ \\
\hline & Lonchoglossa caudifera & $*$ & & \\
\hline & Choeroniscus minor & " & $\cdots$ & $\cdots$ \\
\hline & Lonchophylla mordax & $?$ & 一 & $\cdots$ \\
\hline & Carollia perspicillata & $*$ & $?$ & $\cdots$ \\
\hline & C. brevicauda & - & $\cdots$ & $\cdots$ \\
\hline & Rhinophylla pumilio & $*$ & - & $\cdots$ \\
\hline & R. fischerae & $\overrightarrow{-}$ & $\ddot{*}$ & $\rightarrow$ \\
\hline & Sturnira lilium & $\cdot$ & $\cdots$ & $\cdots$ \\
\hline & S. tildae & 一 & 一 & $\cdots$ \\
\hline & Uroderma magnirostrum & 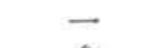 & $\cdots$ & 一 \\
\hline & U. bilobatum & $*$ & 一 & $\cdots$ \\
\hline & Vampyrops helleri & $*$ & 一 & $\cdots$ \\
\hline & Vampyrodes caraccioli & - & - & $\cdots$ \\
\hline & Vampyriscus bidens & - & 一 & $\cdots$ \\
\hline & Chiroderma villosum & 一 & - & $\cdots$ \\
\hline & C. trinitatum & * & - & $\cdots$ \\
\hline & Ectophylla macconelli & - & - & $\cdots$ \\
\hline & Artibeus jamaicensis & - & - & $\cdots$ \\
\hline & A. cinereus & - & 一 & $\cdots$ \\
\hline & A. concolor & • & $\cdots$ & - \\
\hline & A. lituratus & - & * & $\cdots$ \\
\hline & Ametrida centurio & • & $\cdots$ & 一 \\
\hline & Desmodus rotundus & $\cdot$ & 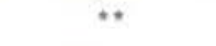 & $\cdots$ \\
\hline & Diaemus youngii & 一 & 一 & $\cdots *$ \\
\hline THYROPTERIDAE & Thyroptera tricolor & $*$ & $\cdots$ & 一 \\
\hline VESPERTILIONIDAE & Myotis nigricans & * & 一 & $\cdots$ \\
\hline & M. albescens & - & - & $\cdots$ \\
\hline & M. simus & - & 一 & $\cdots$ \\
\hline & Eptesicus melanopterus & * & 一 & - \\
\hline & E. brasiliensis & $\bullet$ & - & 一 \\
\hline MOLOSSIDAE & Eumops Trumbulli & * & - & - \\
\hline & Molossus molossus & * & 一 & - \\
\hline & M. ater & $\cdot$ & 一 & 一 \\
\hline TOTAL & 54 ESPECIES & 36 & 17 & 36 \\
\hline
\end{tabular}

Notas preliminares... 
a distribuição das 17 espécies encontradas no Parque. Como já vimos acima, destas 17 espécies, 6 têm ampla distribuição, ocorrendo nas três localidades. Apenas 2 espécies $R$ hinophylla fischerae e Uroderma magnirostrum, foram encontradas exclusivamente no Parque. Das 9 espécies restantes, 8 foram coletadas também em Manaus e apenas 1 existe no Parque e em Belém.

Climaticamente, as três localidades aqui consideradas diferem bastante entre si, havendo maior semelhança entre Manaus e o Parque Nacional da Amazônia (Nimer, 1977). Estas duas localidades apresentam clima úmido com 1 a 2 meses secos; as precipitações pluviométricas são, respectivamente, em torno de $2.200 \mathrm{~mm}$ e $1.750 \mathrm{~mm}$. Em Belém, o clima é superúmido sem seca, com uma precipitação de cerca de $2.500 \mathrm{~mm}$. Estas diferenças climáticas, bem como diferenças geológicas e pedológicas, têm seu reflexo também na vegetação. No Parque Nacional da Amazônia, mais, encontra-se uma floresta caracterizada como mata de cipó (Pires, 1974), com biomassa mediana, grande quantidade de cipós e maior penetração de luz.

Evidentemente, o material disponivel é ainda muito escasso, assim como os conhecimentos sobre como os morcegos se integram à biota local. de tal modo que é ainda prematuro tentar-se qualquer interpretação em termos de "centros de endemismo" (Brown Jr. \& Ab' Saber, 1979)

\section{AGRADECIMENTOS}

$O$ autor agradece ao IBDF, por ter financiado a pesquisa; ao $\mathrm{Eng}^{\circ}$. Florestal Armando P. C. Filho, ao guarda florestal Roberto dos Santos, a Maria Christina Amoroso e à bióloga Lyn Branch que, de alguma forma, contribuíram para este trabalho. Ainda ao Dr. Wal$\operatorname{dir}$ A. Taddei, pelas leituras dos originais.

\section{SUMMARY}

A Study on 'bats was made at the Amazon National Park located partly in the state of Para and partly in Amazonas. This Park has an area of 1.000 .000 ha.
The objectives of the study were to make a survey of the species that exist there and to collect data about their distribution and ecology. Bird nets of 120 to $180 \mathrm{~m}^{2}$ were used to capture the bats; in 10 different places within the accessible area of the Park. One hundred and thirty three individuals of 17 species of 5 families were captured; 11 species from the family Phillostomidae. Carollia is the most common genus. It was concluded that the coexistence of so many different species in a single area is possible because of the diversity in feeding habitat. Frugivorous bats were the most commonly collected but insectivorous, nectivorous, and sanguivorous bats were also found. The bat fauna of Manaus, the National Parque of Amazônia, and Beiem were compared. Only $11 \%$ of the species were found to be in common to the three localities. The fauna of the Park showed greater affinity ic that of Manaus than Belem.

\section{BIBLIOGRAFIA}

BroWN, JR., K.S. \& AB' SÁBER, A.N

1979 - Ice-age forest refuges and evolution in the Neothopics: correlation of paleoclimatological geomorphological and pedological endemism. Univ. Săo Paulo, Inst. Geogr., Paleoclimas 5: 1-30.

Gcodwin, G.G. \& Greenhall, A.M.

1971 - A review of the bats of Trinidad and To. bago, Builetin of the Am. Museum of Natural Hystory. New York, 122 (3): 195-301.

Greenhall, A.M. \& Paradiso, J.L

1968 - Bats and Bat Banding. Bureau of Sport Fisheries and wildlife Resource Publication 72 Washington, D. C. 47p.

HANDleY, C.O.

1967 - Bats of canopy of an Amazonian Forest. Atas do Simpósio sobre a Biota Amazônica. (5) Zoologia, 211-215.

Husson, A.M.

1962 - The bats of Surinam. Leiden, E. J. Brill. $282 p$.

NIMER, E

1977 - Clima. In : Fundaçăo Instituto Brasileiro de Geografia e Estatística. Diretoria Técnica. Geografia do Brasil, V.5. Regiäo Norte. Rio de Janeiro, SERGRAF - IBGE, n. 39-58.

PIRES, J.M

1974 - Tipos de vegetação da Amazônia. Brasil Florestal, 5 (17): 48:58

I B D F

1978 - Plano de Manejo do Parque Nacional da Amazônia (Tacajós), Brasilia, (Mimeografado). 
PIRLOT, P.

1972 - Chiroptères de Moyenne Amazonie. Extrait de Mammalia, 36 (1): 71-85.

TADDEI, U.A.

1973 - Phyllostomidae da Regiāo Norte - Ocidental do Estado de Sâo Paulo. Tese. Fac.

Fil. Ciências e Let. São José do Rio Preto.
VizotTo, L.D. \& TAdDEI, V.A.

1973 - Chave para a determinaçāo de quirópteros brasileiros. Săo José do Rio Preto, Francal. Boletim Ciências. Faculdade de Filosofia e Letras, (1): 1-72p.

REIS, N.R. \& MORK, W.Y.

1979 - Wangiella dermatitides, isolated from bats in Manaus, Brasil. Sabourandia, 17(3):213.

(Aceito para publicação em 05/06/79) 